\title{
Obstrução uretral aguda causada por tumor venéreo transmissível em um cão
}

\author{
Acute Urethral Obstruction Due to Transmissible Venereal Tumor in a Dog \\ Ulisses Nilo Landi' ${ }^{1}$, Talita Bianchin Borges ${ }^{2}$, Ana Maria Quessada ${ }^{3}$, Jessé Lahos Borges', \\ Dhiego Henrique de Oliveira ${ }^{4}$, Melissa Marchi Zaniolo' ${ }^{1}$, Pollyana Linhares Sala' ${ }^{1}$ Rita de Cássia Lima Ribeiro' ${ }^{1}$
}

\begin{abstract}
Background: Transmissible venereal tumor (TVT) is a sexually transmitted, contagious, round cell neoplasm that affects mainly the external genital organs of dogs of both sexes. Canine TVT is practically the only tumor transmitted by cellular transplantation under natural conditions. The tumor occurs in all dog breeds and in various parts of the world, especially in the tropics and subtropics. Sexually active dogs that roam are at increased risk of acquiring the infirmity. The diagnosis is clinical, and confirmed by cytology. The most effective treatment is chemotherapy with vincristine. The aim of this report is describes a case of one male dog with an acute urethral obstruction caused by TVT.

Case: In a Veterinary Medical Teaching Hospital (VMTH), a dog of unknown age was admitted. Volunteers from an animal protection institution rescued the dog from the streets after information from residents. Upon admission in the VMTH, the dog had an acute urethral obstruction, detected on clinical examination. Cystocentesis was performed to relieve the condition. Complete clinical examination and blood count were performed. Hematological examination revealed anemia and thrombocitopeny. The clinical examination revealed a swelling in the preputial area, and penile exposure was not possible. Because of this it was not possible to place the urethral catheter in the animal. However, preputial fistula and a friable, bleeding mass suggestive of a TVT were detected. For penile inspection and correction of the obstruction, the dog was submitted to a surgical procedure with an incision in the preputial midline. After the incision was made, several masses with a friable and bleeding appearance were found. The masses that prevented the urinary flow were removed and, cleansed with a physiological solution. The skin was sutured to restore the normal anatomy. In the same procedure, the dog was neutered. Cytological examination of the masses confirmed the diagnosis of TVT. After the surgery, the animal was treated with vincristine sulfate for three weeks and completely recovered. Due to the findings of the hemogram that were suggestive of hemoparasite, the animal was later referred for clinical investigation.

Discussion: The animal in this case had lived on the streets, where it contracted TVT. This tumor is found most often in dogs that roam. The cystocentesis performed in the patient is used in cases of urethral obstruction in dogs where it is not possible to pass catheter. The friable and bleeding masses with ulceration that were presented by the animal, demonstrated macroscopic aspects characteristic of tumor venereal transmissible. In this type of neoplasm can appear masses in diverse places of the body, but in this dog, the masses were only in the genitalia. The urethral obstruction was caused by the masses around the urethral orifice, and urethral obstructions in dogs may be from neoplasms. The cytological examination confirmed the diagnosis, and such examination is adequate to diagnose TVT. The treatment with chemotherapy is effective to control the disease. However, in this animal, surgical intervention was necessary to restore normal urinary flow. The chemotherapy with vincristine was used after the surgical procedure to eliminate remaining masses. The animal was castrated because of the need for population control in free-living dogs. Although not common, urethral obstruction in dogs can be caused by tumor venereal transmissible.
\end{abstract}

Keywords: canine, neoplasm, obstruction, urethra.

Descritores: canino, neoplasia, obstrução, uretra. 


\section{INTRODUÇÃO}

O Tumor venéreo transmissível (TVT) é uma neoplasia de células redondas, contagiosa, de ocorrência natural em cães, transmitida sexualmente, afetando principalmente a genitália externa com localização preferencial na mucosa genital de ambos os sexos [15].

Em um estudo comprovou-se que o TVT é endêmico em pelo menos 90 países em todos os continentes [19], particularmente em populações de animais ferais e, em algumas regiões, é o tumor mais comum da espécie canina. Ocorre em todas as raças de cães em várias partes do mundo, especialmente em zonas tropicais e subtropicais. Pode acometer outros canídeos como lobos, chacais, coiotes e raposas [4].

O tumor venéreo transmissível canino é praticamente o único tumor transmitido por transplante celular em condições naturais. As células viáveis podem ser transmitidas a animais susceptíveis e transferir-se espontaneamente para outras mucosas, através de pele ou mucosa lesada durante o coito, lambidas, lutas, mordeduras e outras situações [1]. Devido à sua natureza de transmissão por contato sexual, cães sexualmente ativos de vida livre, estão em maior risco de contrair esta doença [9].

A maior parte dos animais afetados apresenta massas na genitália [13,15], sanguinolentas e friáveis [5], proporcionando características únicas à neoplasia. Desta forma, o diagnóstico pode ser clínico, embora deva ser confirmado pela citologia ou histopatologia $[5,11]$.

O tratamento mais efetivo é quimioterápico utilizando-se vincristina [3].

A obstrução uretral em cães representa uma emergência devido à possibilidade de hidronefrose, insuficiência renal aguda e óbito. Pode surgir como consequência de diversas doenças, entre elas neoplasias [12].

\section{CASO}

Foi atendido em um Hospital Veterinário Universitário (HVU) um cão, sem raça definida, idade desconhecida, resgatado das ruas por voluntários de uma ONG de proteção animal, após informação de moradores. Ao dar entrada no HVU o cão apresentava obstrução uretral aguda, tendo sido realizada cistocentese para alívio da condição.

No exame clínico o animal apresentou parâmetros normais, com peso de $13 \mathrm{~kg}$, temperatura $38,8^{\circ} \mathrm{C}$, frequência respiratória $40 \mathrm{mpm}$, frequência cardíaca 112 bpm. Durante o exame clinico foi colhido sangue para exame hematológico e notou-se aumento de volume do prepúcio, com impossibilidade de exposição peniana. Devido a isso, não foi possível colocar sonda uretral no paciente. No entanto, foi detectada fístula prepucial e massas friáveis e sangrantes sugestivas de tumor venéreo transmissível (Figura 1). Para inspeção peniana e correção da obstrução, o cão foi submetido a procedimento cirúrgico com incisão na linha média prepucial (Figura 2). Após a abertura foram visualizadas diversas massas com aspecto friável e sangrante. Foram retiradas as massas que impediam o fluxo urinário, realizou-se limpeza com solução fisiológica e a pele do prepúcio foi suturada restabelecendo-se a anatomia normal. Na ocasião, o cão foi castrado. Após o procedimento realizou-se imprint das massas para exame citológico, que confirmou o TVT. O hemograma foi indicativo de infecção por hemoparasita já que apresentava anemia e trombocitopenia.

Após a cirurgia, iniciou-se o tratamento com sulfato de vincristina durante três semanas e o animal obteve completa recuperação da neoplasia. Devido aos achados do hemograma (anemia e trombocitopenia) que foram sugestivos de hemoparasita, o animal foi encaminhado para investigação clínica.

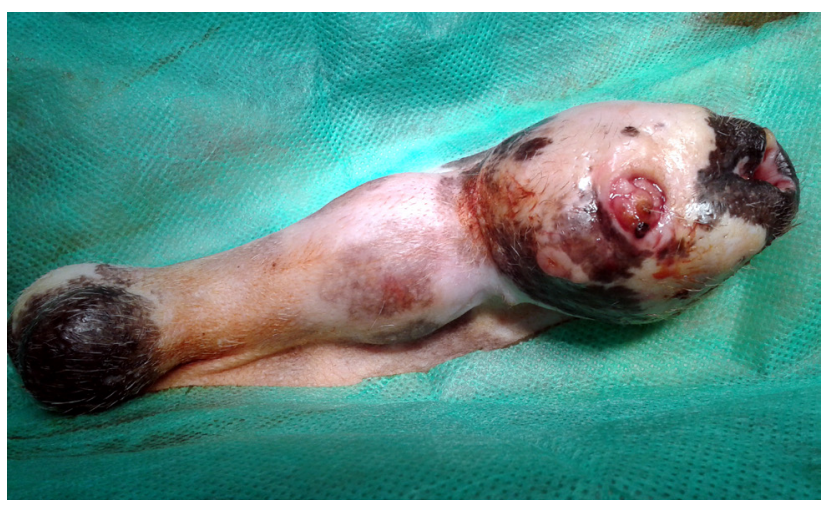

Figura 1. Cão macho, SRD, idade desconhecida apresentando fistula prepucial e massas friáveis e sangrantes sugestivas de tumor venéreo transmissível.

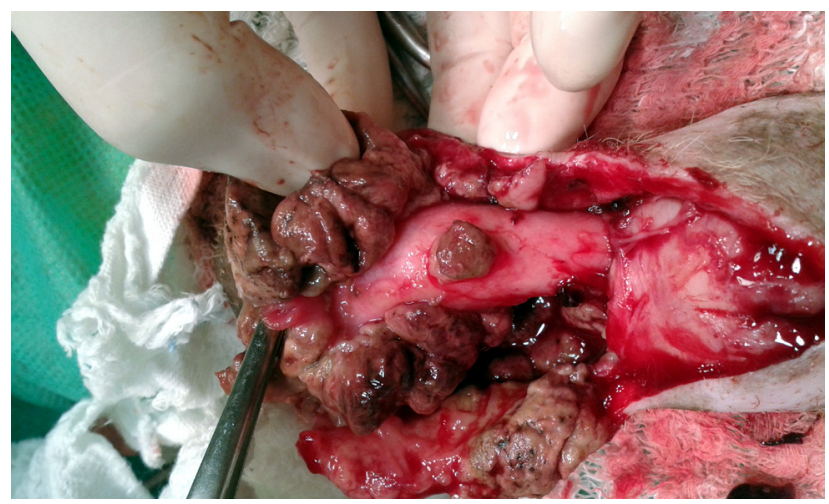

Figura 2. Cão macho, SRD, idade desconhecida sendo submetido a procedimento cirúrgico com incisão na linha média prepucial, para inspeção e retirada de massas tumorais para alívio de obstrução uretral. 
DISCUSSÃO

O cão, objeto deste relato, era um animal de vida livre, não castrado, provavelmente abandonado nas ruas. Possivelmente esta condição foi decisiva para seu contágio com a doença, sendo que o TVT atinge, na maioria das vezes, animais que habitam áreas com alta concentração de cães abandonados $[17,18]$.

A cistocentese realizada quando o paciente deu entrada, pode ser levada a efeito quando o cão apresenta obstrução uretral [7,14], como ocorreu com o paciente em questão.

Em relação à localização a maioria dos cães acometidos por TVT apresentam massas na genitália $[3,5,13]$, embora a neoplasia possa acometer outros locais do corpo menos frequentes como cavidades oral e nasal, conjuntiva, mucosa anal, região glútea, gengiva [10] e glândula mamária [8]. No cão deste relato só havia massas no pênis e prepúcio.

As massas apresentadas pelo animal deste relato mostravam aparência de tumor venéreo transmissível como ulceração com aspecto de couve-flor, sanguinolentas e friáveis. Tais aspectos são característicos do TVT como está registrado por diversos autores $[3,5,13]$.
A obstrução uretral apresentada pelo animal foi causada pela presença das massas ao redor do orifício uretral, impedindo o fluxo urinário normal. Obstruções uretrais em cães podem ser provenientes de neoplasias [12], como no animal aqui estudado.

O exame citológico confirmou o diagnóstico, sendo que tal exame é adequado para diagnosticar neoplasias de células redondas como é o caso do TVT [16].

A quimioterapia com vincristina é eficaz para controlar a doença [16]. No entanto, neste animal, foi necessária a intervenção cirúrgica para restabelecimento do fluxo urinário normal. O tratamento quimioterápico foi realizado para eliminação completa das massas.

O animal foi castrado porque o TVT se dissemina com maior incidência em áreas com maior número de cães SRD, tornando necessário o controle populacional, incluindo castração [6].

Embora não seja comum, TVT pode causar obstrução uretral em cães.

Declaration of interest. The authors report no conflicts of interest. The authors alone are responsible for the content and writing of the paper.

\section{REFERENCES}

1 Amaral A.S., Sandra B.S., Isabelle F., Fonseca L.S., Andrade F.H., Gaspar L.F. \& Rocha N.S. 2007. Cytomorphological characterization of transmissible canine venereal tumor. Revista Portuguesa de Ciências Veterinárias. 102: 253-260.

2 Andrade S.F., Sanches O.C., Gervazoni E.R., Lapa F.A.S. \& Kaneko V.M. 2009. Comparação entre dois protocolos de tratamento do tumor venéreo transmissível em cães. Clínica Veterinária. 82(14): 56-62.

3 Brandão C.V.S, Borges A.G. \& Ranzani J.J.T. 2002. Tumor venéreo transmissível: estudo retrospectivo de 127 casos (1998-2000). Revista de Educação Continuada CRMV-SP. 5: 25-31.

4 Chikweto A., Kumthekar S., Larkin H., Deallie C., Tiwari K.P., Sharma R.N. \& Bhaiyat M.I. 2013. Genital and Extragenital Canine Transmissible Venereal Tumor in Dogs in Grenada, West Indies. Open Journal of Veterinary Medicine. 3: 111-114.

5 Drumond K.O., Quessada A.M., Silva S.M.M.S., Costa F.A.L., Silva L.S., Pinho F.P. \& Lopes R.R.F.B. 2013. Transmissible Venereal Tumor Treated with Autohemotherapy. Acta Scientiae Veterinariae. 41 : 1107.

6 Furini A.A.C., Guimarães P.M., Silva F.S., Lopes M.A.S.M., Castro K.F., Atique T.S.C. \& Atique Netto H. 2014. Estudo da variação neutropênica pelo sulfato de vincristina em cães com TVT tratados no hospital veterinário do noroeste paulista. Arquivos de Ciências Veterinária e Zoologia da Unipar. 17(1): 5-9.

7 Godoi D.A., Regazolli E., Beloni S.E. \& Zanutto M.S. 2011. Urolitíase por cistina em cães no Brasil. Arquivo Brasileiro de Medicina Veterinária e Zootecnia. 63(4): 881-886.

8 Horta R.S., Fonseca, L.S., Miranda D.F.H., Quessada, A.M., Rocha Neto H.J. \& Silva S.M.M.S. 2014. Tumor venéreo transmissível (TVT) com metástase para a glândula mamária. Acta Scientiae Veterinariae. 42(Suppl 1): 1-6.

9 Kabuusu R.M., Stroup D.F. \& Fernandez C. 2010. Risk factors and characteristics of canine transmissible venereal tumours in Grenada, West Indies. Veterinary and Comparative Oncology. 8(1): 50-55.

10 MacLachlan N. J. \& Kennedy P.C. 2008. Tumor of the Genital Systems. In: Meuten D.J. (Ed). Tumor in domestic animals. 4th edn. New Jersey: Wiley-Blackwell, pp.547-573. 
U.N. Landi, T.B. Borges, A.M. Quessada, et al. 2017. Obstrução uretral aguda causada por tumor venéreo transmissível em um cão. Acta Scientiae Veterinariae. 45(Suppl 1): 212.

11 Magalhães A.M., Ramadinha R.R., Barros C.S.L. \& Peixoto P.V. 2001. Estudo comparativo entre citopatologia e histopatologia no diagnóstico de neoplasias caninas. Pesquisa Veterinária Brasileira. 21(1): 23-32.

12 Martins J.I.C. 2014. Obstrução uretral por causas atípicas. 120f. Lisboa. Dissertação (Mestrado em Ciências Veterinárias). Universidade de Lisboa. Faculdade de Medicina Veterinária.

13 Montoya-Flórez L.M. Pedraza-Ordonéz F., Monteiro S. Brandão C.V.S. \& Rocha N.S. 2013. Clinical and cytological staging of transmissible venereal tumour at the Botucatu Veterinary Hospital. Veterinaria y Zootecnía. 7: 75-91.

14 Morishin Filho M.M., Rossetto V.J.V., Brandão C.V.S. \& Ranzani J.J.T. 2010. Uretrolitíase em cão com quatro semanas de idade. Veterinária e Zootecnia. 17(4): 480-484.

15 Murgia C., Pritchard J.K., Kim S.Y., Fassati A. \& Weiss R.A. 2006. Clonal origin and evolution of a transmissible cancer. Cell. 126(3): 477-487.

16 Silva M.C.V., Barbosa R.R., Santos R.C., Chagas R.S.N. \& Costa W.P. 2007. Avaliação epidemiológica, diagnóstica e terapêutica do tumor venéreo transmissível (tvt) na população canina atendida no hospital veterinário da Ufersa. Acta Veterinaria Brasílica. 1(1): 28-32.

17 Sousa J., Saito V., Nardi A.B., Rodaski S., Guérios S.D. \& Bacila M. 2000. Características e incidência do tumor venéreo transmissível (TVT) em cães e eficiência da quimioterapia e outros tratamentos. Archives of Veterinary Science. 5: 41-48.

18 Strakova A. \& Murchison E.P. 2014. The changing global distribution and prevalence of canine transmissible venereal tumor. BMC Veterinary Research. 10(1): 168-178. 